\title{
Recent advances in the JENDL project
}

K. Shibata ${ }^{1, a}$, T. Nakagawa ${ }^{1}$, T. Fukahori ${ }^{1}$, O. Iwamoto ${ }^{1}$, A. Ichihara ${ }^{1}$, N. Iwamoto ${ }^{1}$, S. Kunieda ${ }^{1}$, N. Otuka ${ }^{1}$, J. Katakura $^{1}$, Y. Watanabe ${ }^{2}$, and K. Kosako ${ }^{3}$

1 Nuclear Data Center, Japan Atomic Energy Agency, Tokai, Ibaraki-ken 319-1195, Japan

2 Kyushu University, Kasuga, Fukuoka 816-8580, Japan

3 Shimizu Co., Etchujima, Tokyo 135-8530, Japan

\begin{abstract}
General- and special-purpose JENDL data files are being produced in cooperation with the Japanese Nuclear Data Committee. Nuclear model codes have been developed in order to raise the reliability of the fourth version of JENDL General-Purpose File (JENDL-4). MA and FP data have been evaluated for JENDL-4. The capture cross section of ${ }^{235} \mathrm{U}$ was examined in the energy region from $2 \mathrm{keV}$ to $1 \mathrm{MeV}$. As a follow-up action on the previous library JENDL-3.3, covariances of several nuclei were estimated for a study on ADS. The evaluation for the 2007 versions of JENDL High Energy File (JENDL/HE) and JENDL Photonuclear Data File (JENDL/PD), which are regarded as special-purpose files, is in the final stage. Analyses of neutron transmission experiments reveal the reliability of JENDL/HE.
\end{abstract}

\section{Introduction}

It is 30 years since we released the first version of JENDL general-purpose file (JENDL-1) [1], which was developed in cooperation with the Japanese Nuclear Data Committee (JNDC). We have continued to update general-purpose files and to produce special-purpose files for various applications. After the establishment of the Japan Atomic Energy Agency (JAEA) in 2005, data evaluation for the fourth version of JENDL (JENDL-4) is being performed at the Nuclear Data Center, JAEA. In this new library, much emphasis is placed on improvements of minor actinide (MA) and fission product (FP) data. To achieve this, nuclear model codes have been newly developed. Cross-section covariances were estimated for several nuclei contained in JENDL-3.3 [2]. These covariance data are used for a design study on ADS.

As for special-purpose files, the evaluations for the 2007 versions of JENDL High Energy File (JENDL/HE) and JENDL Photonuclear Data File (JENDL/PD) have been made. Both of the libraries will be released this year.

This paper deals with the recent activities on the developments of JENDL general-purpose and special-purpose files.

\section{Activities for general-purpose file}

\subsection{Development of nuclear model codes}

It is necessary to raise the reliability of MA and FP data in JENDL-4. However, experimental data are scarce for these nuclei. Thus, nuclear model calculations play a significant role in evaluation. Two statistical model codes POD [3] and CCONE [4] have been developed to reflect recent advances

${ }^{a}$ Presenting author, e-mail: shibata.keiichi@jaea.go.jp

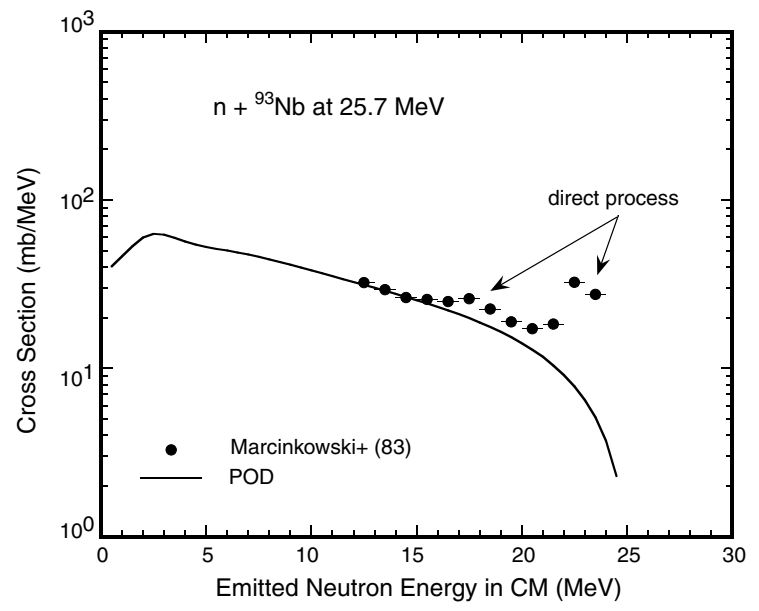

Fig. $1 .{ }^{93} \mathrm{Nb}\left(\mathrm{n}, \mathrm{n}^{\prime}\right)$ at $25.7 \mathrm{MeV}$.

in nuclear theories on evaluation. The POD code is mainly used in the evaluation for medium and medium-heavy nuclei. It consists of the spherical optical model, the one-component exciton model, DWBA, and the Hauser-Feshbach statistical model with width fluctuation corrections, and it is capable of reading transmission coefficients calculated with the coupledchannel optical model. Figure 1 shows the pre-equilibrium neutron emission spectra for the $\mathrm{n}+{ }^{93} \mathrm{Nb}$ reaction calculated with the exciton model. Another example of the POD calculations is illustrated in figure 2, where the calculated capture cross section of ${ }^{209} \mathrm{Bi}$ is compared with experimental data. The spherical neutron optical-model parameters obtained by Koning and Delaroche [5], which are the default values in the program, were used in the calculation. A bump seen around $10 \mathrm{MeV}$ is due to the pre-equilibrium capture, and the calculations reproduce experimental data.

The other code CCONE was developed to evaluate actinide nuclei. It is written in $\mathrm{C}++$ programming language; nuclear properties, decay channels, spectra, etc., are treated 


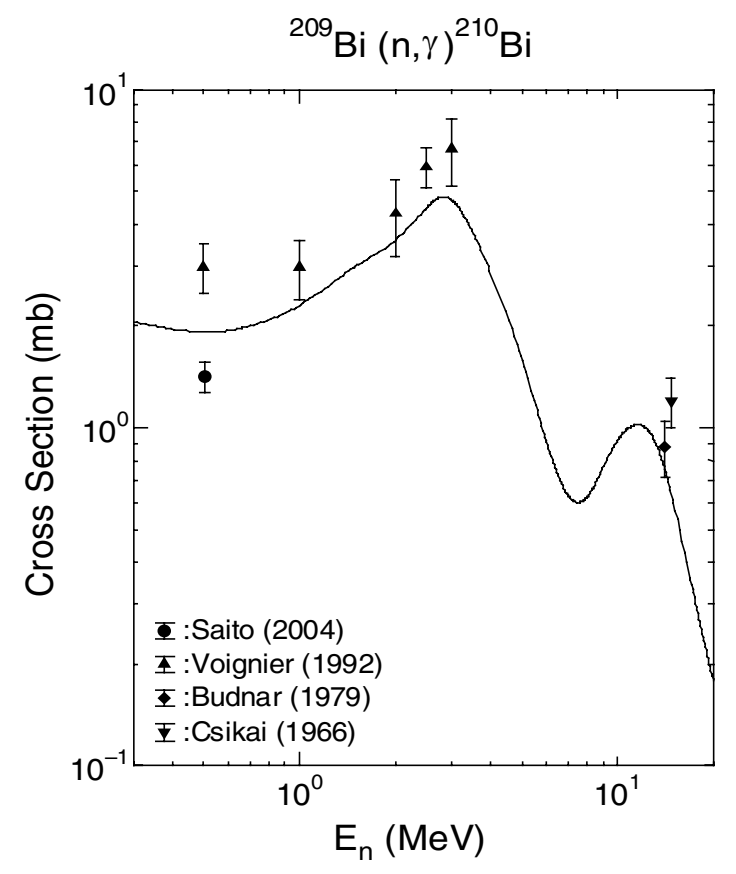

Fig. 2. Capture cross section of ${ }^{209} \mathrm{Bi}$.

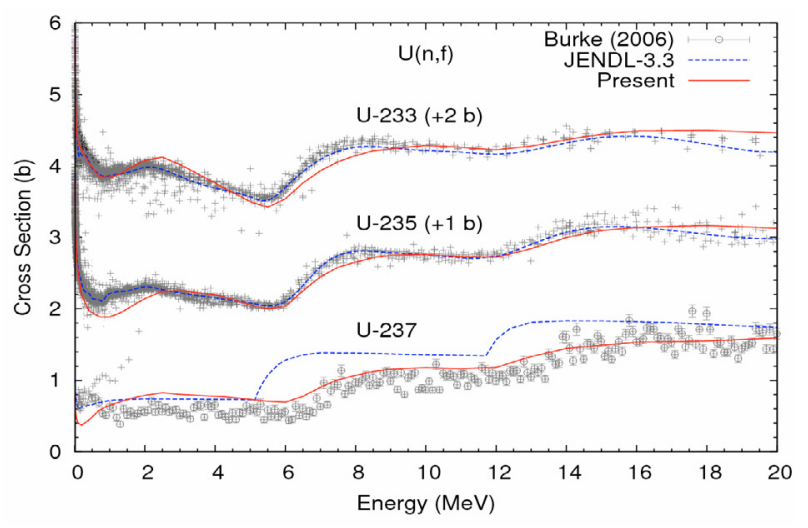

Fig. 3. Fission cross sections of ${ }^{233,235,237} \mathrm{U}$.

as objects. Each object is created dynamically at execution time, which enables one to handle a large number of reaction chains. The CCONE code is based on the coupled-channel optical model, the Hauser-Feshbach theory, and the twocomponent exciton model. The DWBA method is applied to calculate the direct cross sections to excite vibrational levels. A double-humped parabolic barrier [6] is assumed for the fission channel, and all transition states are approximated by a level-density formula. The calculated fission cross sections of ${ }^{233,235,237} \mathrm{U}$ are shown in figure 3, together with JENDL-3.3 and experimental data. In these calculations, we used the dispersive optical model parameters obtained by Soukhovitskii et al. [7], although the deformation parameters were a little bit changed to reproduce measured total cross sections. Fissionbarrier parameters were adjusted so as to fit the measured fission cross sections. The neutron emission spectra from ${ }^{238} \mathrm{U}$ calculated at $14 \mathrm{MeV}$ are illustrated in figure 4 , where the inelastic scattering to vibrational states $\left(1^{-}: 0.6801 \mathrm{MeV}\right.$,

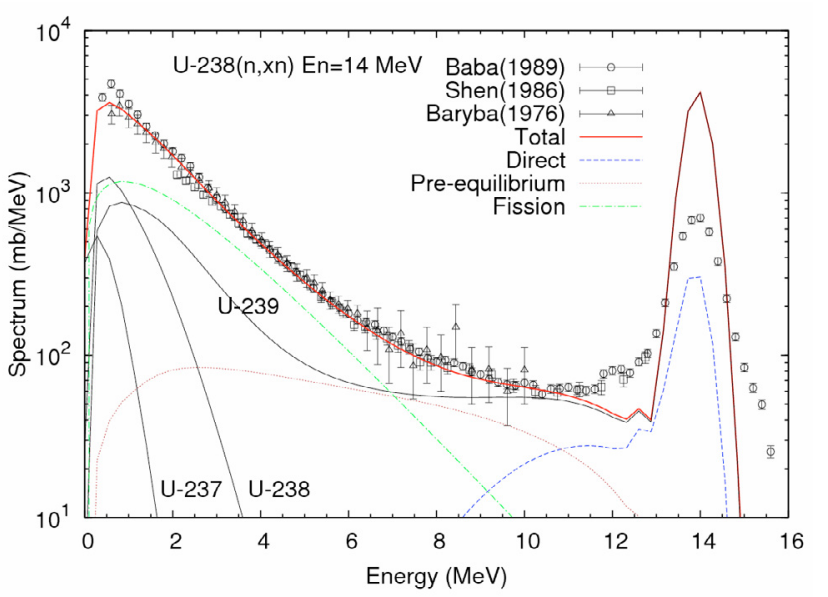

Fig. 4. Neutron emission spectra from ${ }^{238} \mathrm{U}$ at $14 \mathrm{MeV}$.

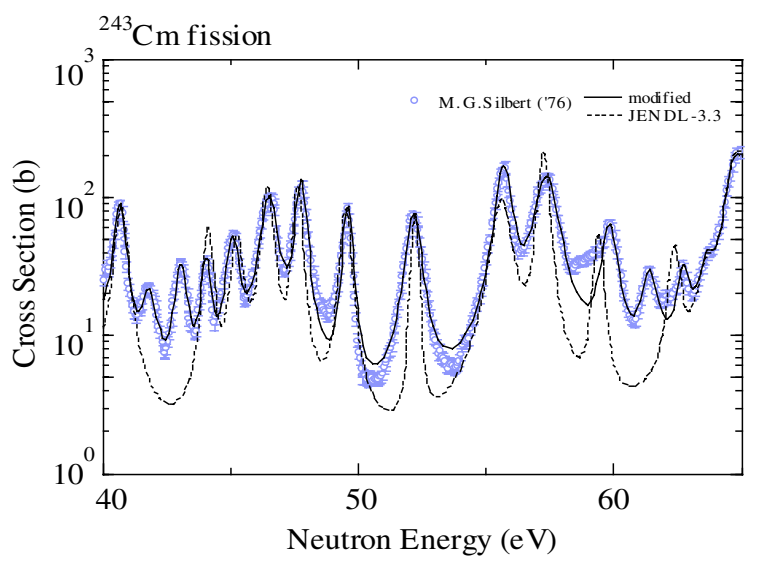

Fig. 5. Fission cross section of ${ }^{243} \mathrm{Cm}$.

$3^{-}: 0.7319 \mathrm{MeV}$ and $2^{+}: 1.0603 \mathrm{MeV}$ ) was estimated by DWBA in addition to the members of the ground-state rotational band. The prompt fission neutron spectra were calculated with the Los Alamos model [8]. Neutrons from multichance fission are included in the calculated spectrum. It is found from the figure that the present calculations are in good agreement with measurements except for the elastic scattering peak at $14 \mathrm{MeV}$. The elastic scattering cross section obtained from integrating the peak (about $800 \mathrm{mb}$ ) measured by Baba et al. [9] is much smaller than the values ( 2 or 3 barns) expected for these actinide nuclei.

\subsection{Actinide data for JENDL-4}

We compiled thermal cross sections of 46 nuclei by considering recent experimental data. Resolved resonance parameters have been updated for 20 nuclei: ${ }^{231,233} \mathrm{~Pa},{ }^{229} \mathrm{Th},{ }^{234,236} \mathrm{U}$, ${ }^{237} \mathrm{~Np},{ }^{236,238,242} \mathrm{Pu},{ }^{242 \mathrm{~g}, 242 \mathrm{~m}} \mathrm{Am},{ }^{242-248} \mathrm{Cm}$ and ${ }^{250,251} \mathrm{Cf}$. As an example, the fission cross section of ${ }^{243} \mathrm{Cm}$ is shown in figure 5 . The presently evaluated data reproduce the experimental data of Silbert [10]. The calculated thermal fission cross section, $587.4 \mathrm{~b}$, agrees with the weighted average of 


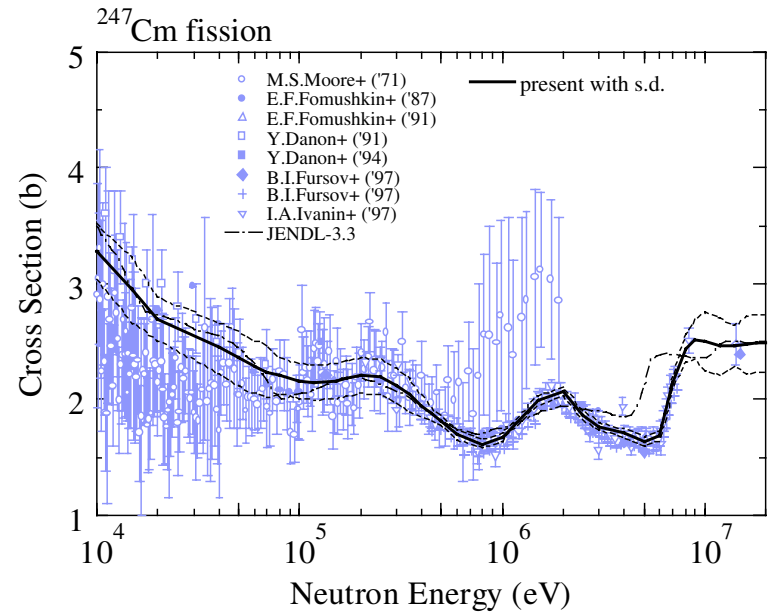

Fig. 6. Fission cross section of ${ }^{247} \mathrm{Cm}$.

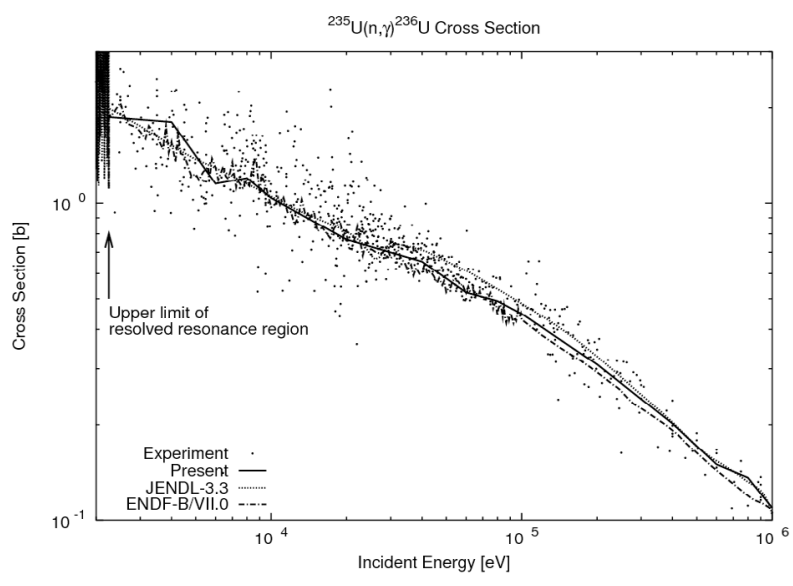

Fig. 7. Capture cross section of ${ }^{235} \mathrm{U}$.

experimental data, $587 \pm 12 \mathrm{~b}$, although Mughabghab [11] recommended a value of $617 \pm 20 \mathrm{~b}$.

The average number of prompt neutrons released per fission was also updated for 8 nuclei: ${ }^{239,240,241,242} \mathrm{Pu}$ and 232,233,235,238 U.

We have performed the evaluation of fission cross sections on the basis of available experimental data. The leastsquares fitting code GMA [12] was used for this purpose. The GMA analyses were performed for 26 nuclei: ${ }^{230,232} \mathrm{Th},{ }^{231} \mathrm{~Pa}$, ${ }_{232,233,234,236,237} \mathrm{U},{ }^{237} \mathrm{~Np},{ }^{236,238,242,244} \mathrm{Pu},{ }^{241,242 \mathrm{~g}, 242 \mathrm{~m}, 243} \mathrm{Am}$, $242,243,244,245,246,247,248 \mathrm{Cm}$ and ${ }^{249,252} \mathrm{Cf}$. Figure 6 shows the evaluated fission cross section of ${ }^{247} \mathrm{Cm}$ above $10 \mathrm{keV}$. In this evaluation, not only cross sections but also covariances are determined.

Another important GMA analysis has been performed [13] for the capture cross section of ${ }^{235} \mathrm{U}$ in the energy region above $2 \mathrm{keV}$. This was undertaken to improve the $\mathrm{C} / \mathrm{E}$ values of criticalities for the fast-neutron assembly BFS with $\mathrm{UO}_{2}$ fuels. The result is shown in figure 7 . It is found from the figure that the present re-evaluation is smaller than JENDL3.3, but it is still somewhat larger than ENDF/B-VII.0 [14] above $100 \mathrm{keV}$. The difference between the present work and ENDF/B-VII.0 should be clarified by a further investigation.

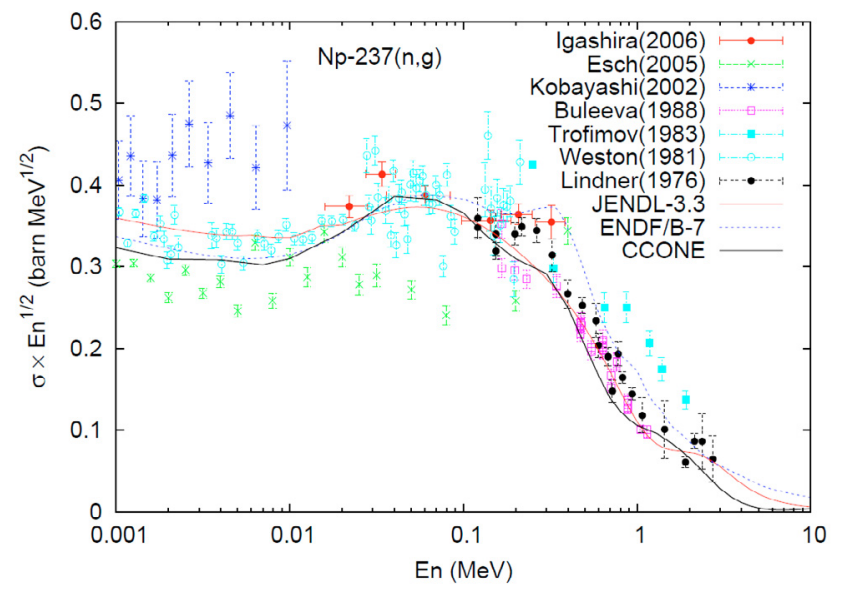

Fig. 8. Capture cross section of ${ }^{237} \mathrm{~Np}$.

The criticalities of BFS cores were calculated using these revised cross sections and sensitivities to the capture cross sections of JENDL-3.3. The results are given in table 1. The present re-evaluation of ${ }^{235} \mathrm{U}$ capture cross section improves the criticality prediction by about $0.2 \%$. Further improvement is expected by re-examining the capture cross section in the resolved resonance region down to $100 \mathrm{eV}$.

In the non-resonance energy region, the CCONE code, which was mentioned above, was used to evaluate smooth cross sections of 21 nuclei: ${ }^{237} \mathrm{~Np},{ }^{236-242,244} \mathrm{Pu}$, $241,242 \mathrm{~g}, 242 \mathrm{~m}, 243 \mathrm{Am}$ and ${ }^{242-248,250} \mathrm{Cm}$. Figure 8 shows the capture cross section of ${ }^{237} \mathrm{~Np}$. The dispersive coupled-channel optical model parameters, which were mentioned in section. 2.1 , were used in the calculation.

Table 1. Criticalities (C/E) of BFS cores.

\begin{tabular}{ccc}
\hline Core & JENDL-3.3 & Revised \\
\hline $62-1$ & 0.9900 & 0.9923 \\
$62-3 \mathrm{~A}$ & 0.9934 & 0.9951 \\
\hline
\end{tabular}

\subsection{FP and medium-weight nucleus data for JENDL-4}

Resolved resonance parameters of JENDL-3.3 FP nuclei were examined for JENDL-4 by taking account of recent measurements. As a result, the parameters for 107 nuclei were updated. Moreover, the parameters for additional 13 nuclei were newly evaluated for JENDL-4. The calculated thermal cross sections and resonance integral were compared with the compilation of Mughabghab [11]. As for ${ }^{99} \mathrm{Tc}$, we obtained a thermal value of $23.6 \pm 0.7 \mathrm{~b}$ for the capture cross sections from latest measurements, and revised the parameters for a negative resonance.

In the non-resonance energy region of FP nuclei, nuclear model calculations are performed to evaluate cross sections. Although many parameters are required as input to nuclear model codes, the most important ones are optical model parameters. The optical model parameters were searched for by using the coupled-channel optical model code OPTMAN [15]. Systematics of neutron and proton optical model parameters 


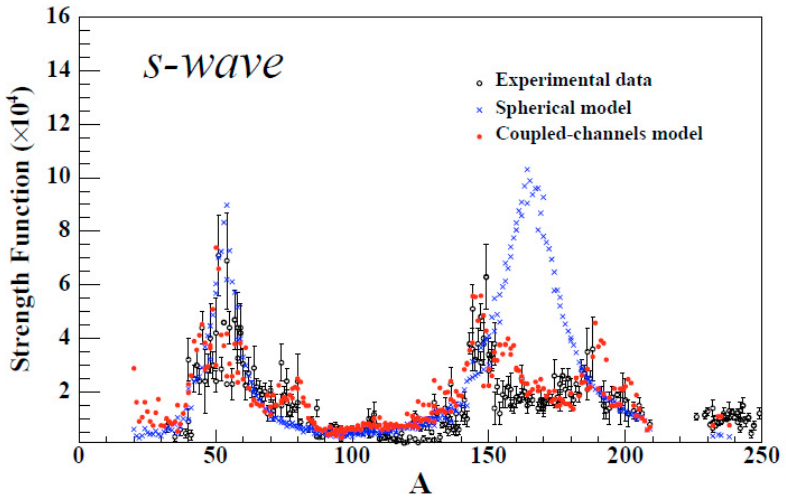

Fig. 9. Calculated s-wave strength functions at $10 \mathrm{keV}$, together with experimental data.

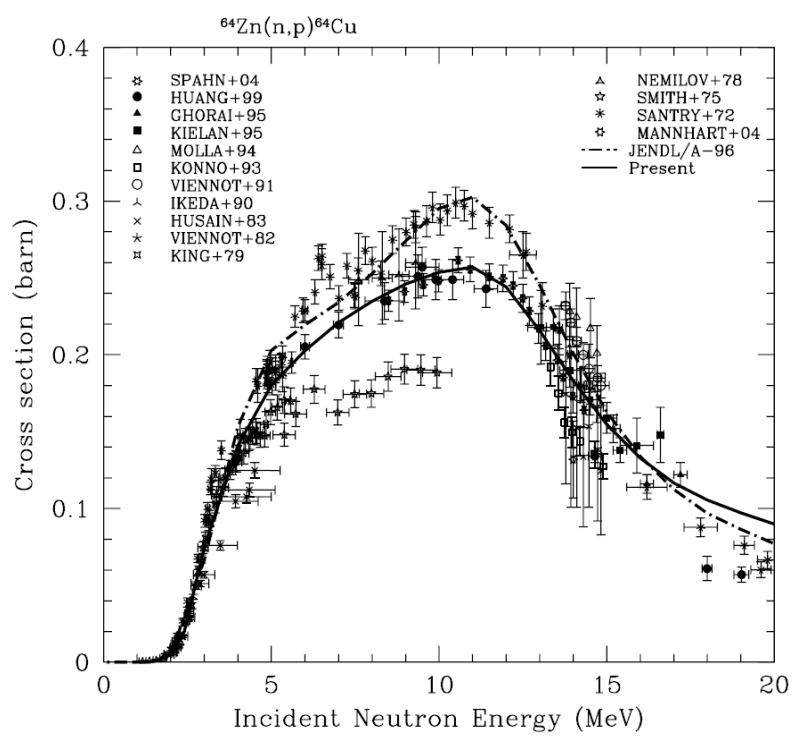

Fig. 10. (n,p) reaction cross section of ${ }^{64} \mathrm{Zn}$.

were obtained [16] by comparing with experimental data on $\mathrm{Fe}, \mathrm{Ni}, \mathrm{Cu}, \mathrm{Zr}, \mathrm{Nb}, \mathrm{Mo}, \mathrm{Sn}, \mathrm{W}, \mathrm{Au}, \mathrm{Pb}, \mathrm{Bi}$, Th and $\mathrm{U}$ in the energy region from $1 \mathrm{keV}$ to $200 \mathrm{MeV}$. In order to check the low-energy behaviour of the optical model parameters, we calculated neutron strength functions at $10 \mathrm{keV}$, which represent average resonance properties. As seen in figure 9, the present calculations are in good agreement with experimental data. On the other hand, the spherical optical model cannot reproduce the experimental data in the mass region from $\mathrm{A}=150$ to 200. Evaluations of $\mathrm{Zn} \mathrm{[17]} \mathrm{and} \mathrm{Nd}$ isotopes were performed by using the optical model parameters thus obtained. For example, figures 10 and 11 show the calculated (n,p) cross section of ${ }^{64} \mathrm{Zn}$ and $\gamma$-ray emission spectra for elemental $\mathrm{Zn}$, respectively.

As for medium-weight nuclei, the cross sections of $\mathrm{Ca}$ isotopes were evaluated [18], since $\mathrm{Ca}$ is an ingredient of concrete and its neutron cross sections are important from the viewpoint of nuclear engineering. In this evaluation, the statistical-model code TNG [19] was applied to calculate neutron-induced reaction cross sections by using the spherical neutron optical-model parameters obtained by Koning and Delaroche [5]. The (n,p) cross section of ${ }^{42} \mathrm{Ca}$ is illustrated in

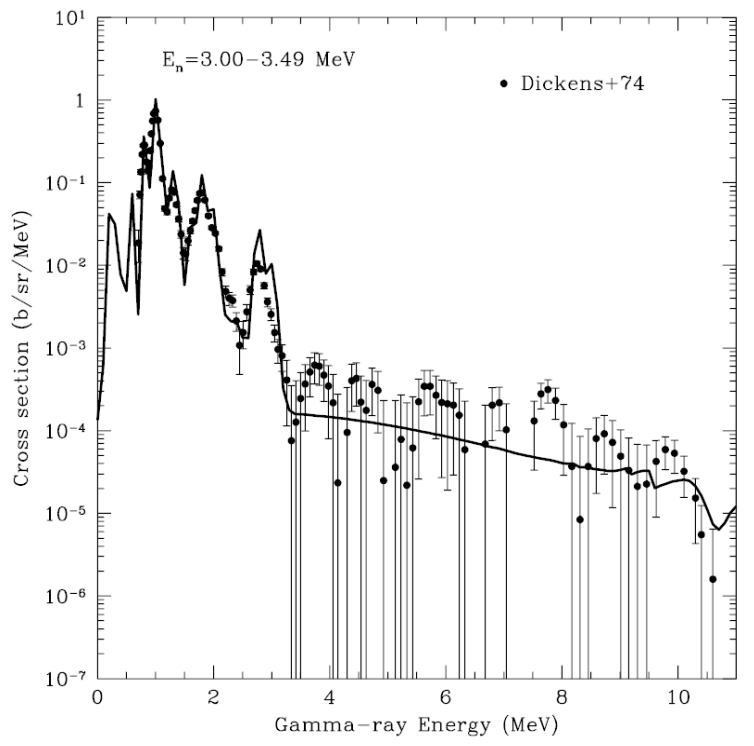

Fig. 11. $\gamma$-ray emission spectra for elemental $\mathrm{Zn}$ at 125 degrees.

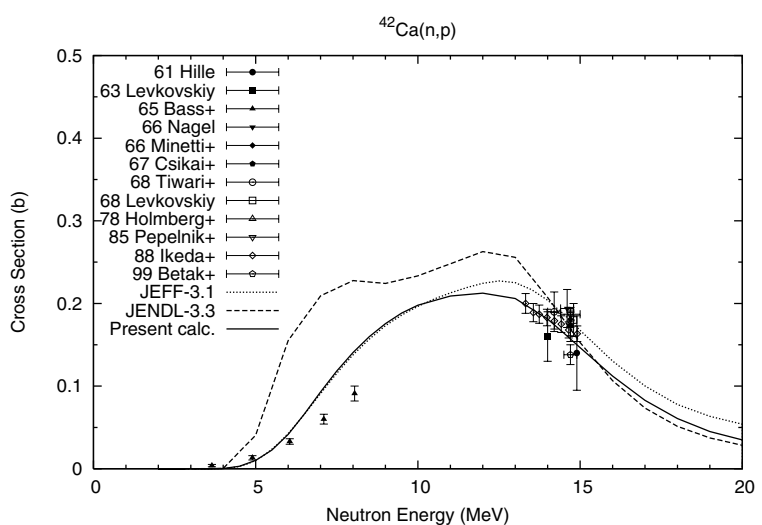

Fig. 12. (n,p) cross section of ${ }^{42} \mathrm{Ca}$.

figure 12. The cross-section shape of JENDL-3.3 is different from those of the present evaluation and JEFF-3.1 [20].

\subsection{Covariances of JENDL-3.3 for ADS}

JENDL-3.3, which was released in 2002, contains covariance data for important 20 nuclei. However, more covariance data are required by those who are involved in the development of ADS. We evaluated [21,22] covariances of the elastic scattering cross section of ${ }^{15} \mathrm{~N}$, inelastic scattering cross sections of ${ }^{206,207,208} \mathrm{~Pb}$, and ${ }^{209} \mathrm{Bi}$, and fission and capture cross sections of ${ }^{237} \mathrm{~Np},{ }^{238} \mathrm{Pu},{ }^{241,242 \mathrm{~m}, 243} \mathrm{Am}$ and ${ }^{243} \mathrm{Cm}$ contained in JENDL-3.3. For actinide nuclei, covariances of resolved resonance parameters were also estimated together with those of $v_{\mathrm{p}}$ for ${ }^{237} \mathrm{~Np}$ and ${ }^{241,243} \mathrm{Am}$. These covariances were obtained by analyzing the experimental data on which JENDL3.3 was based or by using the uncertainties in the parameters required as input to nuclear model calculation. Figure 13 shows the standard deviations of the ${ }^{244} \mathrm{Cm}(\mathrm{n}, \mathrm{f})$ cross sections which were estimated by using the least-squares fitting code GMA [12]. 


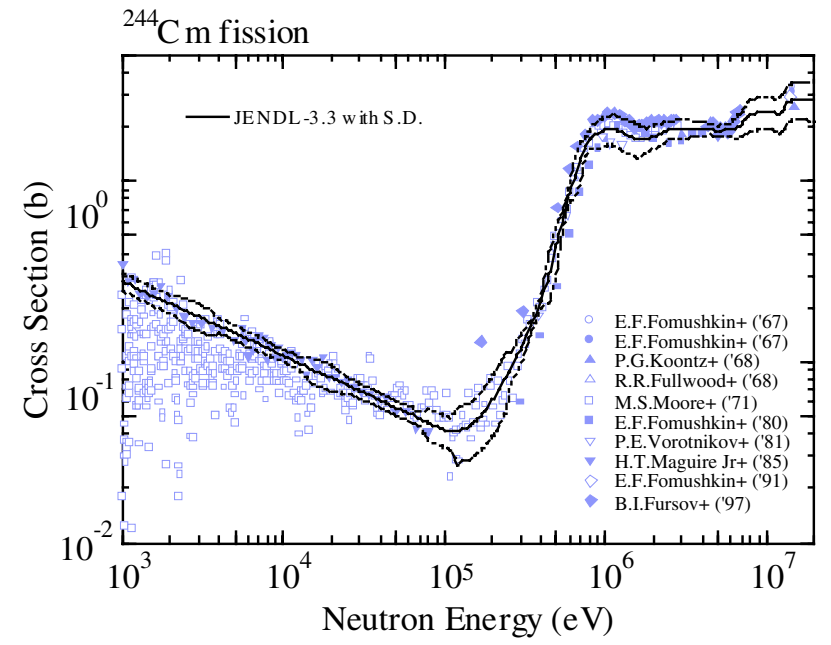

Fig. 13. Fission cross sections of ${ }^{244} \mathrm{Cm}$. The dashed lines stand for the standard deviations obtained in the present work.

\section{Activities for special-purpose file}

\subsection{JENDL High Energy file}

JENDL High Energy file has been developed for applications such as ADS, shielding, RI production, radiation damage, activation, and dosimetry. The first version was released as JENDL/HE-2004 [23] in 2004. It includes neutron- and proton-induced reaction data for 66 nuclei up to $3 \mathrm{GeV}$. In the energy region below $200 \mathrm{MeV}$, the conventional statistical model and direct-interaction methods were used while the intra nuclear cascade model or quantum molecular dynamics was employed above $200 \mathrm{MeV}$. Data of 25 nuclei were updated for the 2007 version, JENDL/HE-2007. We have newly evaluated high-energy data of 32 nuclei: ${ }^{19} \mathrm{~F},{ }^{23} \mathrm{Na}$, ${ }^{35,37} \mathrm{Cl},{ }^{36,38,40} \mathrm{Ar},{ }^{92,94-98,100} \mathrm{Mo},{ }^{181} \mathrm{Ta},{ }^{197} \mathrm{Au},{ }^{204,206-208} \mathrm{~Pb}$, ${ }^{209} \mathrm{Bi},{ }^{235,238} \mathrm{U},{ }^{237} \mathrm{~Np},{ }^{238-242} \mathrm{Pu},{ }^{241,242 \mathrm{~g}, 242 \mathrm{~m}} \mathrm{Am}$. Therefore, JENDL/HE-2007 is supposed to include about 100 nuclei for neutron- and proton-induced reactions. The high-energy fission cross section of ${ }^{238} \mathrm{U}$ is shown in figure 14 .

Benchmark tests of the evaluated high energy data were performed by using MCNPX [24]. Experimental data on thick-target neutron yields (TTY) and neutron transmission were used as benchmarks. The TTY data were measured [25] at LANL for 113 and $256 \mathrm{MeV}$ protons. The neutron transmission experiments were performed [26] at TIARA using $\mathrm{p}+{ }^{7} \mathrm{Li}$ neutrons at $43 \mathrm{MeV}$ and $68 \mathrm{MeV}$. Figure 15 shows a comparison of the calculated neutron transmission for $100 \mathrm{~cm}$ thick iron shield with the measurements obtained with $68-\mathrm{MeV}$ protons at TIARA. It is found from the figure that the JENDL/HE-2004 calculations are much better than those of LA-150 [27] and NRG2003 [28] in the entire energy region.

\subsection{JENDL photonuclear data file}

Photonuclear data are important for accelerator shielding, radiation therapy and non-destructive assay. The photoabsorption below $150 \mathrm{MeV}$ mainly takes place via excitation to the

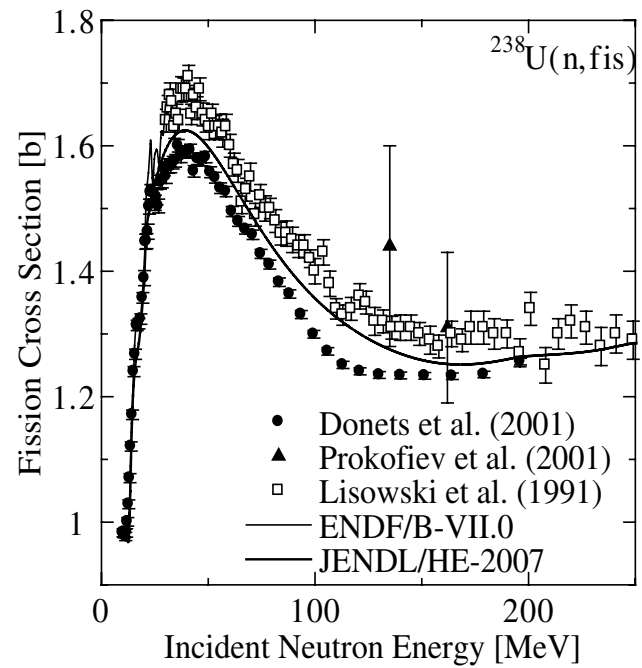

Fig. 14. Fission cross section of ${ }^{238} \mathrm{U}$.

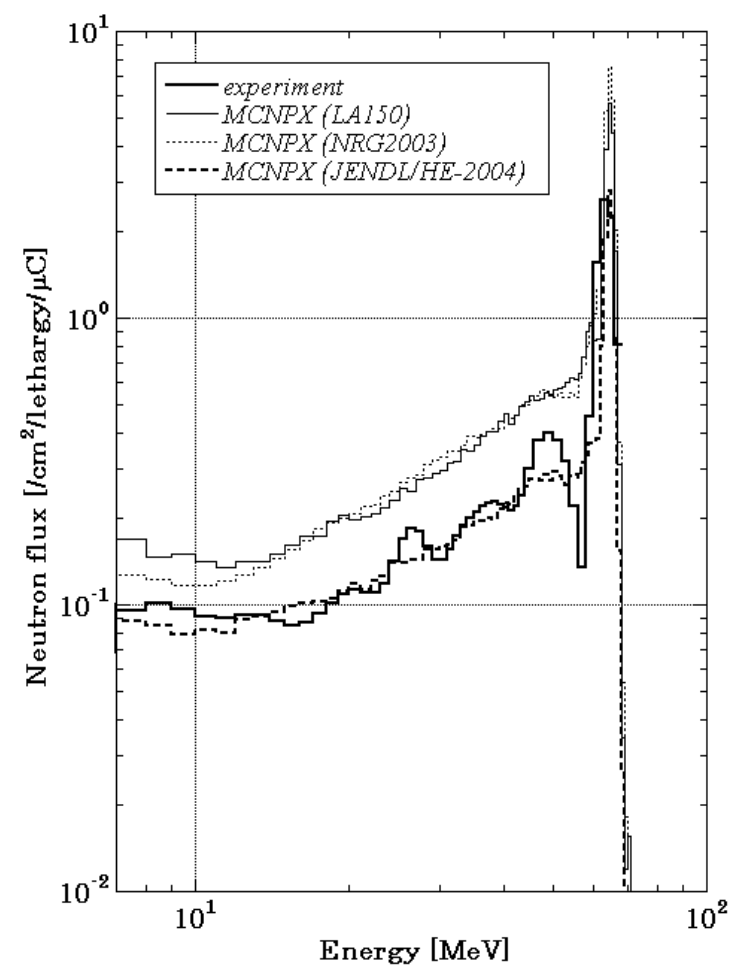

Fig. 15. Comparison of calculated neutron transmission for $100-\mathrm{cm}$ thick iron shield with measurements for neutrons produced by $\mathrm{p}+{ }^{7} \mathrm{Li}$ at $68 \mathrm{MeV}$.

giant electric dipole resonance (GDR) or via disintegration of a photon-neutron pair in a nucleus. The latter process is called the quasideuteron model (QDM). Therefore, the photoabsorption cross section can be expressed by a sum of these two processes. We evaluated photonuclear data by using the evaporation model codes ALICE-F [29] and MCPHOTO [30]. The 2004 version was already released as JENDL/PD-2004 [31], which contained data for 68 nuclei from ${ }^{2} \mathrm{H}$ to ${ }^{237} \mathrm{~Np}$. Figure 16 shows the photo-neutron emission cross section of 


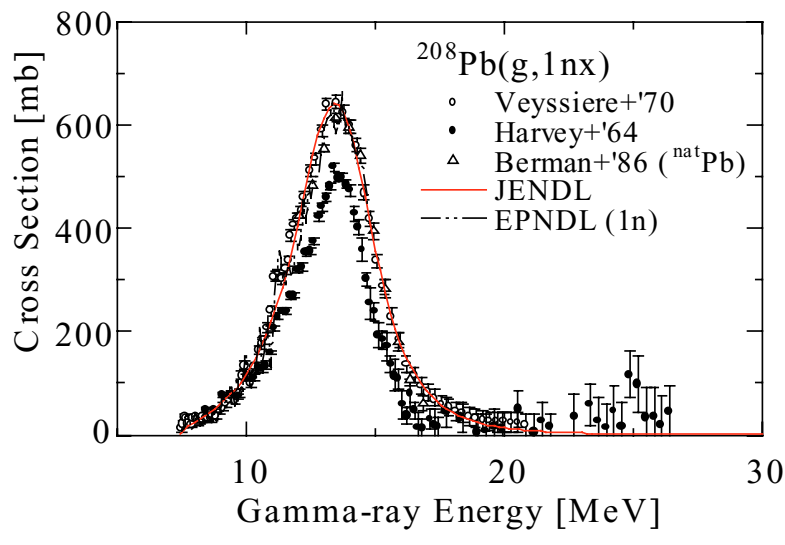

Fig. 16. Single-neutron emission cross section of ${ }^{208} \mathrm{~Pb}$.

${ }^{208} \mathrm{~Pb}$, as an example. After the release, data on 6 nuclei were updated: ${ }^{3} \mathrm{He},{ }^{12} \mathrm{C},{ }^{55} \mathrm{Mn},{ }^{181} \mathrm{Ta}$, and ${ }^{181,184} \mathrm{~W}$. Moreover, the data for 107 nuclei were taken from the photonuclear data file [32] produced at KAERI. As a result, the 2007 version contains the data for 175 nuclei in the photon energy up to $150 \mathrm{MeV}$.

\section{Concluding remarks}

We are developing general- and special-purpose JENDL data files. The general-purpose library JENDL-4 is being produced as a mid-term project at JAEA. In the new library, it is necessary to raise the reliability of MA and FP data. To achieve this, two nuclear model codes were developed and global coupledchannel optical model parameters were obtained in the FP region. Data for a lot of actinide nuclei were re-evaluated in the resonance and smooth regions. Moreover, the capture cross section of ${ }^{235} \mathrm{U}$ was revised in the energy region from $2 \mathrm{keV}$ to $1 \mathrm{MeV}$, although a follow-up action may be needed to resolve the problem of the sodium-voided reactivities for the BFS fastneutron cores with $\mathrm{UO}_{2}$ fuels.

JENDL High Energy File (JENDL/HE-2007), which contains neutron- and proton-induced reaction data of about 100 nuclei up to $3 \mathrm{GeV}$, and Photonuclear Data File (JENDL/PD2007), which contains photon-induced reaction data of 175 nuclei up to $150 \mathrm{MeV}$, are almost ready for release. It was found that the calculations using JENDL/HE reproduce measured neutron transmission very well.
The authors would like to thank the members of the Japanese Nuclear Data Committee for their efforts to perform data evaluations and benchmark testing.

\section{References}

1. S. Igarasi et al., JAERI 1261 (1979).

2. K. Shibata et al., J. Nucl. Sci. Technol. 39, 1125 (2002).

3. A. Ichihara et al., JAEA-Data/Code 2007-012 (2007).

4. O. Iwamoto, J. Nucl. Sci. Technol. 44 (in press).

5. A.J. Koning, J.P. Delaroche, Nucl. Phys. A 713, 231 (2003).

6. S. Bjørnholm, J.E. Lynn, Rev. Mod. Phys. 52, 725 (1980).

7. E.Sh. Soukhovitskii et al., Phys. Rev. C 72, 024604 (2005).

8. D.G. Madland, J.R. Nix, Nucl. Sci. Eng. 81, 213 (1982).

9. M. Baba et al., JAERI-M 89-143 (1989).

10. M.G. Silbert, LA-6239-MS (1976).

11. S.F. Mughabghab, Atlas of Neutron Resonances (Elsevier, The Netherlands, 2006).

12. W.P. Poenitz, in Proceedings of Conference on Nuclear Data Evaluation Methods and Procedures, BNL, 1980 (BNL-NCS51363, 1981), p. 249.

13. N. Otuka et al., J. Nucl. Sci. Technol. 44 (in press).

14. M.B. Chadwick et al., Nucl. Data Sheets 107, 2931 (2006).

15. E.Sh. Soukhovitski et al., JAERI-Data/Code 2005-002 (2005).

16. S. Kunieda et al., J. Nucl. Sci. Technol. (in press).

17. N. Iwamoto, J. Nucl. Sci. Technol. 44 (in press).

18. K. Shibata, J. Nucl. Sci. Technol. 44, 10 (2007).

19. C.Y. Fu, ORNL/TM-7042 (1980).

20. A. Koning et al., JEFF Report 21 (OECD/NEA, 2006).

21. T. Nakagawa, J. Nucl. Sci. Technol. 42, 984 (2005).

22. K. Shibata, T. Nakagawa, J. Nucl. Sci. Technol. 44, 1 (2007).

23. Y. Watanabe et al., in Proceedings of the International Conference on Nuclear Data for Science and Technology, Santa Fe, 2004 (AIP, 2005), p. 326.

24. L.S. Waters, LA-UR-02-2607 (2002).

25. M.M. Meier et al., Nucl. Sci. Eng. 104, 339 (1990).

26. H. Nakashima et al., Nucl. Sci. Eng. 124, 243 (1996).

27. M. Chadwick et al., Nucl. Sci. Eng. 131, 293 (1999).

28. A.J. Koning et al., in Proceedings of the International Conference on Nuclear Data for Science and Technology, Santa Fe, 2004 (AIP, 2005), p. 422.

29. T. Fukahori (private communication).

30. N. Kishida, H. Kadotani (private communication).

31. N. Kishida et al., in Proceedings of the International Conference on Nuclear Data for Science and Technology, Santa Fe, 2004 (AIP, 2005), p. 199.

32. M. Chadwick et al., International Atomic Energy Agency Report, IAEA-TECDOC-1178 (2000). 